\title{
Performance Analysis of Underwater Optical Wireless Communication Systems Based on CD-Optical OFDM and Various Subcarriers Indexes
}

\author{
Monther Noaman Hasan ${ }^{1}$, Ahmed Ghanim Wadday ${ }^{2}$, Faris M. Ali ${ }^{2}$ \\ ${ }^{1}$ Department of Communications Techniques Engineering \\ Engineering Technical college- Najaf. \\ Al-Furat Al-Awsat Technical University, Najaf-Iraq \\ datasun2000@yahoo.com \\ ${ }^{2}$ Department of communications Techniques engineering \\ Engineering Technical college- Najaf. \\ Al-Furat Al-Awsat Technical University, Najaf-Iraq \\ ahmadghw@atu.edu.iq \\ ${ }^{2}$ Department of Communications Techniques Engineering \\ Engineering Technical college- Najaf. \\ Al-Furat Al-Awsat Technical University, AL- Najaf 31001-Iraq \\ faris@atu.edu.iq
}

\begin{abstract}
The speedy growing of communication technology and visual services, larger capacity and higher-speed transmissions are required in contemporary underwater applications, which produce the present aquatic systems finally flop to meets requirements. A recently emerging technique in the scope of wireless communication is underwater optical wireless communications (UOWC). Due to using an optical signal, we can achieve a high data rate (approximately Gbps) over medium communication link ranges because of the harsh environment in the underwater channel where optical signals are faced many difficulties in transmission such as absorption and scattering. So, the main problem in the UOWC system performance is the short link range. In this paper, a DPSK with Coherent Detection CD-Optical OFDM with a various number of OFDM subcarriers has been designed and simulated under high turbulence water. Concerning their bit error data rate (BER) achievements, the comparing bit error rate impose is corresponding to $10^{-5}$. The simulation results show the achieved communication distances by the suggested system for deferent OFDM subcarriers indexes (128, 256,512 and 1024 ) are $3.9 \mathrm{~m}, 4.8 \mathrm{~m}, 5 \mathrm{~m}$, and $5.9 \mathrm{~m}$ respectively. It is clear when we increase the number of OFDM subcarriers the link rage will be increased linearly due to the combating of the inter-symbol interference (ISI) effect. The design and simulation have been accomplished using the optisystem ${ }^{\mathrm{TM}}$ tool.
\end{abstract}

Keywords - Underwater Optical Wireless Communications (UOWC), Differential phase shift keying (DPSK), Coherent Detection (CD), Orthogonal Frequency Division Multiplexing OFDM, optisystem ${ }^{\mathrm{TM}}$ tool.

\section{Introduction}

About 73 percent of our earth's surface is wrapped by water and by the rapid technological advances, the area of underwater communications has grown rapidly and widely with comprehensive applications in trade and martial systems. Applications such as far-off monitoring in the marine oil production, pollution control, scientific oceanographic data collection, disaster detection, sensor networks for monitoring climate changes, coral reefs, autonomous drifter swarms, power plants, marine archaeology and rescue missions and early warning, national security [1]. The investigation into different wireless communication techniques in the underwater has played the most significant part in the ocean and other aquatic environments [1]. Contrary to terrestrial wireless communication, underwater wireless channels are influenced by intense conditions such as nautical milieu, restricted power, clatter, and bandwidth[2]. Thus the underwater wireless channel is the greatest complicated and tough due to many circumstances such as absorption, scattering, multipath transmission, dispersion, restricted bandwidth, and power sources. While the underwater systems confronted these exclusive circumstances, numerous recent deviances, which have not been faced in terrestrial wireless communication systems, emerge for future underwater wireless networks in Radiofrequency, acoustic and optical communication systems. Acoustics and optical, because of the possibility of extended distance of transmission and high-bandwidth network communications in dimensions, modems with power- 
equipped and automated systems are among these challenges. The theme of underwater wireless networks has enticed and increase the interest of investigators, not only from the academe moreover in the martial and industrial fields, based on their attractive and unique elements as well as the potential advantages of advanced underwater communications. In later years, a great deal of research has been done on underwater wireless networks, but further developments on the wireless system underwater continue to be a problem, given the previous challenges posed by the exploitation of acoustic and optical wireless channels. There are three possible methods in underwater communications [2]. RF by sending electromagnetic waves, acoustic by sending sound waves and optical communication by sending optical waves. Each of these techniques has advantages and limitations. Optical waves are electromagnetic waves between $400 \mathrm{~nm}$ (blue light) and $700 \mathrm{~nm}$ (red light) of wavelength. Optical signals is characterized by high speed, extremely short wavelength making it capable of communicating at extremely high speeds (Beyond the 1Gbps). The optical carriers used in wireless communication, though, are usually extremely short because of strong water absorption and strong backscatter of suspended particles in the optical frequency band[3]. The author in [4] proposed and experimentally demonstrated an Intensity Modulation/Direct Detection Orthogonal Frequency Division Multiplexing (IM/DD) OFDM based underwater optical wireless communication system. The transmitter was a single compact blue LED and the receiver was a low-cost PIN photodiode. The Quadrature Amplitude Modulation (QAM) modulation with different order and subcarriers was used to evaluate the underwater optical link and bit error rate. The transmission link was $40 \mathrm{~m}$ and performed in coastal water with negligible scattering effect. The experimental results showed that the bit data rates of $227.8 \mathrm{Mb} / \mathrm{s}$ at a Bit Error data Rate (BER) of $1.4610^{-3}$ using 16-QAM and $232.95 \mathrm{Mb} / \mathrm{s}$ at a BER of $3.2810^{-3}$ using 32-QAM respectively at $2 \mathrm{~m}$ link range. Over a $2 \mathrm{~m}$ underwater channel, the bit data rates were $162.46 \mathrm{Mb} / \mathrm{s}$ using 16QAM, 167.31 Mb/s using 32-QAM, and 138.07 Mb/s using 64-QAM respectively. In [5] proposed an underwater video transmission system with the laser link. The channel performance was verified by changing the tank visibility. By using a tank of $4.6 \mathrm{~m}$ the experiment procedure was accomplished. The distance between the transmitter and the receiver was $5 \mathrm{~m}$. The transmitter laser diode was $488 \mathrm{~nm}$ and has optical power $50 \mathrm{mw}$. They concluded that the underwater video transmission utilizing the underwater laser signal is achievable, even at a visibility range of 4 times visibility. Hassan M. Oubei and et al [6] presented a high-speed underwater wireless optical communication using non-return-to-zero on-off keying (NRZ-OOK) modulation scheme. The experiment was tested in a $7 \mathrm{~m}$ water pool. The transmitter was a $520 \mathrm{~nm}$ laser diode (LD) with $1.2 \mathrm{GHz}$ bandwidth and an avalanche photodiode (APD) module as the receiver. The result of the measured BER from the experiment was $2.23 \times 10^{-4}$.In [7] proposed and experimentally examined the repetition coding OFDM
(RC - OFDM), Alamoutii-OOFDM and MISO OOFDM systems in high turbulence water, The outcomes validate the diversity gain of MIMO and that the Alamoutii-OOFDM system is further unaffected by delay than the RC - OFDM system.

In this paper, we have proposed a differential phase shift (DPSK) using coherent detection-Optical orthogonal frequency division multiplexing (CD-Optical OFDM) and examine the proposed system with various number of OFDM subcarriers $(128,256,512$ and 1024) to find out the performance optimization such as BER and transmission range of the UOWC system under turbid conditions.

The rest of the paper is organized as follows. The key principles are described in Sect. 2. The system design is described in Sect. 3. In Sect. 4 channel modeling is described. In Sect. 5. The simulation results are demonstrated the. Finally, conclusions are discussed in Sect. 6.

\section{Theoretical Principles}

In this section we will demonstrate the key theoretical principles such as OFDM technique and optical coherent detection.

\section{A. OFDM}

Nowadays, OFDM is considered always as a strong candidate to use in most systems. OFDM is a type of multicarrier modulation (MCM) in which a data flux at one rate is split into many parallel lower rate streams, the streams are separately modulated onto different frequency carriers' waves, or subcarriers, for transmission over the same channel. OFDM has been applied only recently to optical communication, however, in many optical systems including wireless optical, single-mode fiber, multimode optical fiber, and plastic optics, there is a growing quantity of documents on the hypothetical and practical act of OFDM which is considered an amended copy of Frequency Division Multiplexing (FDM) [8]. OFDM uses many carriers per a given spectrum that are very close to each other, however, they remain orthogonal at an exact distance of each other. The use of the Fast Fourier Transform (FFT) and Inverse FFT aid to demodulate and construct the earliest signal until if there is interference between the subcarriers. OFDM signals are generated from a summation of sinusoids, with each matching to a subcarrier. Subcarriers can be mathematically represented as [9]:

$$
\begin{aligned}
& H_{k}(t) \\
& =\left\{\begin{array}{cc}
\sin (2 \pi k \Delta f t) & 0<t<T=1,2,3, \ldots . . N \\
0 & \text { otherwise }
\end{array}\right\}
\end{aligned}
$$

Where $\Delta f$ is the subcarrier spacing, $N$ is the number of subcarriers, and $T$ is the data symbol period. Since the highest frequency component is $N \Delta f$, the transmission bandwidth is approximately $N \Delta f$. Signals are orthogonal if they are mutually independent of each other. These 
